\title{
Evolutionary Neurocontrol: A Novel Method for Low-Thrust Gravity-Assist Trajectory Optimization
}

\author{
Ian Carnelli* \\ ESA, 2001 AZ Noordwijk, The Netherlands

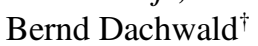 \\ DLR, German Aerospace Center, 82234 Oberpfaffenhofen, Germany \\ and \\ Massimiliano Vasile \\ University of Glasgow, Glasgow, Scotland G12 8QQ, United Kingdom \\ DOI: $\underline{10.2514 / 1.32633}$
}

\begin{abstract}
The combination of low-thrust propulsion and gravity assists to enhance deep-space missions has proven to be a remarkable task. In this paper, we present a novel method that is based on evolutionary neurocontrollers. The main advantage in the use of a neurocontroller is the generation of a control law with a limited number of decision variables. On the other hand, the evolutionary algorithm allows one to look for globally optimal solutions more efficiently than with a systematic search. In addition, a steepest-ascent algorithm is introduced that acts as a navigator during the planetary encounter, providing the neurocontroller with the optimal insertion parameters. Results are presented for a Mercury rendezvous with a Venus gravity assist and for a Pluto flyby with a Jupiter gravity assist.
\end{abstract}

\section{Nomenclature}

$\hat{\mathbf{e}}_{r} \quad=\quad$ sun-spacecraft unit vector

$g_{0}=$ Earth's standard gravitational acceleration

$I_{\mathrm{sp}} \quad=$ specific impulse

$\mathrm{J}=$ fitness function

$\dot{m}_{p}=$ propellant mass flow

$\mathrm{N}^{p}=$ network function

$\mathcal{R}=$ rotation matrix

$\mathrm{S}=$ spacecraft steering strategy

$\mathbf{T}=$ thrust vector

$\hat{\mathbf{t}}=$ thrust unit vector

$\mathbf{u}=$ spacecraft control vector

$v_{\infty}=$ hyperbolic excess velocity

$\mathbf{x}_{\mathrm{s} / \mathrm{c}}=$ spacecraft state $\left(\mathbf{r}_{\mathrm{s} / \mathrm{c}}, \mathbf{v}_{\mathrm{s} / \mathrm{c}}\right)$

$\gamma \quad=$

$\Delta \quad=$ sphere of influence scaling factor

thrust clock angle

aiming point distance on the $B$ plane

thrust cone angle

orbit inclination

gravitational constant

number of reproductions

chromosome/individual

population of chromosomes/individuals/pilots

network internal parameters vector

throttle factor $[0,1]$

evolutionary neurocontroller
Received 5 June 2007; revision received 6 October 2008; accepted for publication 8 October 2008 . Copyright $\odot 2008$ by the authors. Published by the American Institute of Aeronautics and Astronautics, Inc., with permission. Copies of this paper may be made for personal or internal use, on condition that the copier pay the $\$ 10.00$ per-copy fee to the Copyright Clearance Center, Inc., 222 Rosewood Drive, Danvers, MA 01923; include the code 0731-5090/09 \$10.00 in correspondence with the CCC.

${ }^{*}$ Systems Engineer, Advanced Concepts Team, European Space Research and Technology Centre, Keplerlaan 1; Ian.Carnelli@esa.int. Member AIAA.

${ }^{\dagger}$ Scientist; currently Professor, Aachen University of Applied Sciences, Aerospace Engineering Department, Hohenstaufenallee 6, 52064 Aachen, Germany. Member AIAA.

${ }^{+}$Lecturer, Aerospace Engineering Department. Member AIAA.

\author{
Subscripts \\ $\mathrm{pl}=$ assisting planet \\ $1=$ before the gravity-assist maneuver \\ $2=$ after the gravity-assist maneuver \\ Superscript \\ * $\quad=$ optimal
}

\section{Introduction}

G RAVITY assists (GAs) have proven to be the key to interplanetary high-energy missions. They not only make missions feasible that would otherwise be impossible due to large propellant mass fractions, but flybys also make missions more interesting for the scientific community. Additionally, low-thrust (LT) propulsion systems make interplanetary missions more efficient and more flexible, allowing larger launch windows [1]. Hence, the combination of low-thrust propulsion and gravity assists (LTGA) provides an excellent means to reduce propellant mass-fraction requirements.

However, the design of such trajectories is no trivial task. The spacecraft control function on low-thrust arcs is a continuous function of time, and therefore the dimension of the solution space is infinite. The problem is further complicated by considerations of the planet's phasing, especially when multiple gravity assists are sought. Finally, preliminary analysis tools such as the Tisserand plane or Lambert's method are not applicable to LT trajectories.

The complexity of the solution of a multiple-LTGA (MLTGA) problem derives from the complexity of the simpler multiplegravity-assist (MGA) problem. It should be noted that this complexity is not simply due to the hybrid nature of the MLTGA problem. It can be easily shown that even formulating the MGA problem in a homogenous fashion, with continuous decision variables as in the fixed-sequence case, it retains its inherent complexity [2]. This is due to the following reasons: the MGA problem presents a high number of local minima, this number grows with the number of gravity maneuvers, the number of minima further increases if multiple revolutions or deep-space maneuvers are inserted between two subsequent planetary encounters, and the most interesting solutions are generally nested (i.e., their basin of attraction is narrow 
and generally falls within or near a wider basin of attraction of a worse solution).

Some of these reasons are related to the mathematical formulation of the problem and can be mitigated with an appropriate approach. For example, if no deep-space maneuvers are considered and the GA maneuvers are modeled through a powered-swingby model, it can be proved that the MGA problem is solvable incrementally in polynomial time, with a small exponent, through a simple branchand-prune procedure [3] .

Others are instead intrinsically related to the physical nature of the problem. In fact, in a MGA trajectory, the outgoing leg from a GA maneuver is highly sensitive to the incoming conditions. This sensitivity narrows down the size of the basin of attraction in comparison with a direct transfer. Furthermore, the ratio between the orbital periods of the planets tends to destroy any periodicity or symmetry in the solution space.

Despite what could seem intuitive, adding low-thrust arcs to a MGA trajectory does not change the global nature of the solution space. The main reason is that low-thrust arcs (in particular, when the solution is optimal) are only locally shaping each trajectory leg, tuning the entry conditions to a GA maneuver at a lower cost than deep-space maneuvers. This is true unless a multispiral trajectory is inserted between two gravity maneuvers. In this case, low-thrust arcs increase the complexity of the problem. Furthermore, if deep-space maneuvers and an accurate gravity-assist model are considered, the number of possible states for an MGA trajectories grows exponentially with the number of GA maneuvers, even for a fixed sequence. The MLTGA problem therefore inherits the complexity of the MGA problem and adds the local solution of an optimal control problem.

Traditionally, the design and analysis of interplanetary LTGA trajectories undergoes three main steps:

1) The main objectives are selected and the sequence of flyby bodies is outlined.

2) Possible preliminary trajectories are analyzed.

3) The optimal trajectory is calculated.

The first two steps, typically carried out by an expert in trajectory optimization, generate the initial guess for the third optimization phase. Unfortunately, this approach is typically quite inefficient, as each individual problem has to be solved from scratch and many solutions have to be explored before convergence of the optimization method is achieved. Finally, even if a solution is obtained, in most cases it represents a local optimum far from the global optimal solution. Recent studies have attacked the problem using either deterministic (such as branch and prune) or stochastic global optimization methods (such as evolutionary algorithms) [4-8].

Though the use of stochastic-based methods allows the treatment of high-dimensional problems that are otherwise intractable with deterministic problems, modeling the control law through either an indirect approach or through direct collocation could not be the most efficient choice in the preliminary phase of the design.

It is proposed here to use evolutionary neurocontrollers (ENCs) instead, which have proven to be able to generate very good solutions to quite complex problems in an effective and efficient way. The ability of ENCs to find, for example, optimal solar photonic assist trajectories to reach the outer solar system with a solar sail was demonstrated by Dachwald [9]. The proposed ENCs are very flexible because they perform a broad search of the solution space without any special requirement on the regularity of the optimization function and of the constraints. They also allow one to accommodate the cooptimization of additional problem parameters (e.g., launch date, hyperbolic excess velocity, etc.).

If we consider the usual classification of the methods for trajectory design [10], the evolutionary neurocontroller would fall in the class of direct approaches in particular, because the dynamics here are propagated forward in time, in the class of direct shooting methods. This basic classification, however, does not help to understand the essence of the evolutionary neurocontroller. The main difference with respect to the other direct methods is that the neurocontroller generates a time-dependent control law, as for indirect methods, with a low number of decision variables. The advantage can be understood through a simple example: If a multispiral trajectory were to be designed and the number of spirals were not known a priori, a direct shooting methods based on a collocation of the controls would require an increase of the number of decision variables as the number of spirals increases to have a good resolution of the control profile. A neurocontroller would instead require a constant, and small, number of control variables. We can say that as neural networks can be used to map a generic nonlinear function, the neurocontroller can also be used to map a generic controller.

In this paper, we present a novel method to design LTGA trajectories that is based on ENCs. This new tool is an extension of InTrance (Intelligent Trajectory Optimization Using Neurocontroller Evolution), the global LT trajectory-optimization tool developed by Dachwald [11].

\section{Simulation Models}

Some general assumptions are used throughout this paper to simplify the models and to relax the computational effort:

1) Along a low-thrust arc, the spacecraft is subject to the gravity attraction of the sun and to the control acceleration of the engine only. Gravity-assist maneuvers are inserted between two low-thrust arcs as hyperbolic trajectories in the planetocentric reference frame. Finally, along the gravity-assist hyperbola, the spacecraft is subject to the gravity attraction of the GA planet only.

2) The magnitude and direction of the spacecraft's thrust vector can be achieved instantaneously.

3) The spacecraft systems (e.g., solar arrays, electric thrusters, etc.) do not degrade over time.

InTrance implements several solar sail models, two solar electric propulsion (SEP) systems (NASA's NSTAR thruster and QinetiQ's T6 thruster), and a nuclear electric propulsion (NEP) system. The preliminary results presented in this paper make use of the NEP system for the Pluto flyby mission and the (NSTAR) SEP system for the Mercury rendezvous mission.

\section{A. NEP System Model}

In this model, the maximum thrust $\mathbf{T}_{\max }$ and the specific impulse $I_{\mathrm{sp}}$ are assumed to be fixed. The maximum propellant mass flow rate $\dot{m}_{p \text {, max }}$ (required to generate $\mathbf{T}_{\text {max }}$ ) is

$$
\dot{m}_{p, \max }=\frac{\left|\mathbf{T}_{\max }\right|}{\left|\mathbf{v}_{e}\right|}=\frac{\left|\mathbf{T}_{\max }\right|}{I_{\mathrm{sp}} g_{0}}
$$

where $\mathbf{v}_{e}$ is the exhaust velocity and $g_{0}$ is Earth's standard gravitational acceleration. A throttle factor $0 \leq \chi \leq 1$ is used to control the propellant mass flow rate, so that

$$
\dot{m}_{p}(\chi)=\chi \dot{m}_{p, \max }
$$

and

$$
T(\chi)=\chi T_{\max }=\chi \dot{m}_{p, \max } I_{\mathrm{sp}} g_{0}
$$

where $T=|\mathbf{T}|$. Thus, propellant mass flow rate and thrust vary only with $\chi$. In contrast to SEP systems, the thrust is independent of solar distance, which makes the NEP especially suited for missions to the outer solar system. Using the thrust unit vector $\hat{\mathbf{t}}$ to denote the thrust direction, Eq. (3) then becomes

$$
\mathbf{T}(\chi)=\chi T_{\max } \hat{\mathbf{t}}=\chi \dot{m}_{p, \max } I_{\mathrm{sp}} g_{0} \hat{\mathbf{t}}
$$

\section{B. SEP System Model}

The key parameter for a SEP system is the input power $P_{\mathrm{PPU}}$ that is available to the power processing unit (PPU). This power is proportional to that delivered by the solar arrays $P_{\mathrm{SA}}$ (which is $\sim 1 / r^{2}$, where $r$ represents the solar distance). The available power $P_{\mathrm{AV}}$ must also take into account the power required to operate the spacecraft's systems $P_{\text {SYS }}$ : 


$$
P_{\mathrm{AV}}(r)=P_{\mathrm{SA}}\left(\frac{1}{r^{2}}\right)-P_{\mathrm{SYS}}
$$

For the NSTAR engine, a throttle $0 \leq \chi \leq 1$ is used to control the PPU power input, so that $P_{\mathrm{PPU}}$ is defined as

$$
P_{\mathrm{PPU}}(\chi, r)= \begin{cases}0 & \text { if } \chi P_{\mathrm{AV}}(r)<P_{\min } \\ \chi P_{\mathrm{AV}}(r) & \text { if } P_{\min } \leq \chi P_{\mathrm{AV}}(r) \leq P_{\max } \\ P_{\max } & \text { if } \chi P_{\mathrm{AV}}(r)>P_{\max }\end{cases}
$$

where $P_{\min }$ and $P_{\max }$ represent the minimum and maximum power at which the propulsion system can operate (respectively, 0.5 and $2.0 \mathrm{~kW}$ ). According to Williams and Coverstone-Carroll [12], the following polynomial approximation for the propellant mass flow rate (in milligrams/second) and thrust (in millinewtons) can be used in the power range $P_{\min } \leq P_{\mathrm{PPU}} \leq P_{\max }$ :

$$
\begin{gathered}
\frac{\dot{m}_{p}(\chi, r)}{(1 \mathrm{mg} / \mathrm{s})}=0.74343+\frac{0.20951 P_{\mathrm{PPU}}}{(1 \mathrm{~kW})}+\frac{0.25205 P_{\mathrm{PPU}}^{2}}{\left(1 \mathrm{~kW}^{2}\right)} \\
\frac{T(\chi, r)}{(1 \mathrm{mN})}=-3.4318+\frac{37.365 P_{\mathrm{PPU}}}{(1 \mathrm{~kW})}
\end{gathered}
$$

The minimum and maximum mass flow rate and thrust are then

$$
\begin{array}{clrl}
\dot{m}_{p, \min } & =0.9112 \mathrm{mg} / \mathrm{s} & \dot{m}_{p, \max }=2.1707 \mathrm{mg} / \mathrm{s} \\
T_{\min } & =15.251 \mathrm{mN} & T_{\max }=71.298 \mathrm{mN}
\end{array}
$$

Finally, the expression for the specific impulse is

$$
I_{\mathrm{sp}}(\chi, r)=\frac{|\mathbf{T}(\chi, r)|}{\dot{m}_{p}(\chi, r) g_{0}}
$$

\section{Equations of Motion for EP Spacecraft}

When a spacecraft employs chemical thrusters to generate the required $\Delta \mathbf{V}$, the maneuvers can be considered to be impulsive, due to the high level of thrust and consequently short burning times. However, if a spacecraft uses electric propulsion, the burning times are comparable with the spacecraft's orbital period, and hence the thrust has to be included within the equations of motion. Therefore, the differential equations system is

$$
\dot{\mathbf{r}}=\mathbf{v} \quad \dot{\mathbf{v}}=-\frac{\mu}{r^{2}} \hat{\mathbf{e}}_{r}+\frac{\mathbf{T}}{m_{\mathrm{SC}}}
$$

where $\hat{\mathbf{e}}_{r}$ is the unit vector pointing from the attracting body $\mathcal{B}$ toward the spacecraft, and $\mu=G M_{\mathcal{B}}$ is the gravitational constant. Using Eq. (4), one gets for NEP

$$
\ddot{\mathbf{r}}=\chi \frac{T_{\max }}{m_{\mathrm{SC}}} \hat{\mathbf{t}}-\frac{\mu}{r^{2}} \hat{\mathbf{e}}_{r}
$$

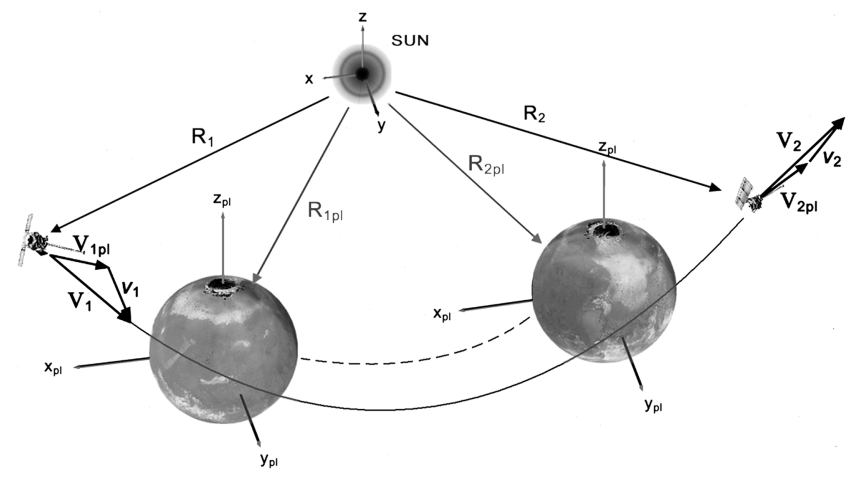

Fig. 1 Schematic diagram of the gravity-assist maneuver.

$$
\dot{m}_{\mathrm{SC}}=-\chi \dot{m}_{p, \max }
$$

whereas for SEP spacecraft,

$$
\begin{gathered}
\ddot{\mathbf{r}}=\frac{T(\chi, r)}{m_{\mathrm{SC}}} \hat{\mathbf{t}}-\frac{\mu}{r^{2}} \hat{\mathbf{e}}_{r} \\
\dot{m}_{\mathrm{SC}}=-\dot{m}_{p}(\chi, r)
\end{gathered}
$$

The orientation of the thrust unit vector $\hat{\mathbf{t}}$ (expressed by the thrust clock angle $\gamma$ and the thrust cone angle $\delta$ ) and the throttle $\chi$ constitute the spacecraft's control vector: that is, $\mathbf{u}=\mathbf{u}(\gamma, \delta, \chi)$.

\section{Gravity-Assist Model}

Labunsky et al. [13] proposed an analytical method that allows an approximate computation of gravity assists. We have implemented this method into InTrance to avoid direct numerical integration of the equations of motion within the assisting body's sphere of influence (SOI). This way, the required computation time is reduced considerably. Nevertheless, this method provides a quite accurate description of the gravity-assist maneuver.

Given the spacecraft state $\mathbf{x}_{\mathrm{sc}}=\left(\mathbf{r}_{\mathrm{sc}}, \mathbf{v}_{\mathrm{sc}}\right)$ on the SOI before the gravity-assist maneuver, the purpose of this method is to provide the spacecraft state on the planet's SOI after the gravity assist. The spacecraft's position and velocity in the heliocentric reference system $\left\{\mathbf{R}_{1}, \mathbf{V}_{1}\right\}$ when entering the SOI, as well as those of the planet $\left\{\mathbf{R}_{\mathrm{pl}, 1}, \mathbf{V}_{\mathrm{pl}, 1}\right\}$, are supposed to be known (see Fig. 1). According to the SOI approximation, the coordinate system is changed into the planetocentric reference frame. The spacecraft's state thus becomes

$$
\begin{aligned}
& \mathbf{r}_{1}=\mathbf{R}_{1}-\mathbf{R}_{\mathrm{pl}, 1} \\
& \mathbf{v}_{1}=\mathbf{V}_{1}-\mathbf{V}_{\mathrm{pl}, 1}
\end{aligned}
$$

where $\left|\mathbf{r}_{1}\right|=R_{\mathrm{SOI}, \mathrm{pl}}$, the subscript 1 indicates a variable before the gravity-assist maneuver, and the subscript $\mathrm{pl}$ is used to indicate a variable of the assisting planet. From the planetocentric point of view, the spacecraft approaches from infinity with a nonzero velocity. Therefore, the trajectory is represented as hyperbolic, and the outbound velocity and position vectors result from a rotation around the angular momentum vector $\mathbf{h}_{\mathrm{sc}}$ with respect to the planet (see Fig. 2). The outbound state of the spacecraft is then obtained using

$$
\begin{aligned}
& \left(\begin{array}{l}
x_{2} \\
y_{2} \\
z_{2}
\end{array}\right)=\mathcal{R}(\phi)\left(\begin{array}{l}
x_{1} \\
y_{1} \\
z_{1}
\end{array}\right) \\
& \left(\begin{array}{l}
v_{x_{2}} \\
v_{y_{2}} \\
v_{z_{2}}
\end{array}\right)=\mathcal{R}(\varphi)\left(\begin{array}{l}
v_{x_{1}} \\
v_{y_{1}} \\
v_{z_{1}}
\end{array}\right)
\end{aligned}
$$

where $\phi$ is the rotation angle of the position vector, $\varphi$ is the rotation angle of the velocity vector, and $\mathcal{R}$ is a rotation matrix. The complementary angles are given by

$$
\phi=\pi+\varphi-2 \gamma \quad \varphi=2 \delta=2 \arctan \frac{\mu}{\Delta v_{\infty}^{2}}
$$

where

$$
\gamma=\pi-\arccos \frac{\mathbf{r}_{1} \cdot \mathbf{v}_{1}}{\left|\mathbf{r}_{1}\right|\left|\mathbf{v}_{1}\right|}
$$

and $v_{\infty}=\sqrt{v_{1}^{2}-2 \mu / r_{1}}$ represents the hyperbolic excess velocity. Finally, $\Delta$ is the aiming point distance defined as the distance between the center of the planet and the inbound asymptote: 


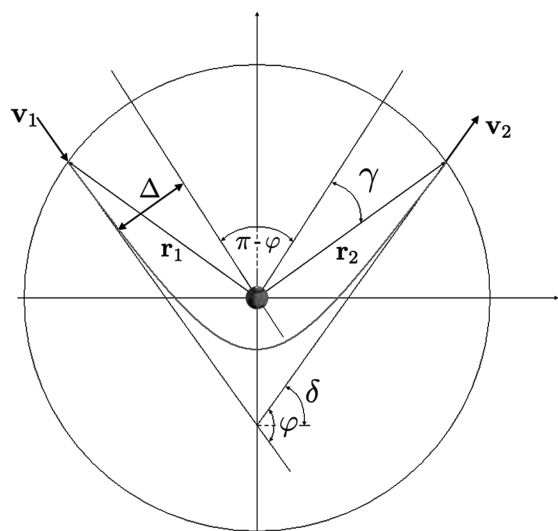

Fig. 2 Schematic diagram of the gravity-assist maneuver.

$$
\Delta=R_{\mathrm{SOI}, \mathrm{pl}} \sin \gamma
$$

Of course, $\Delta$ must be chosen so that the trajectory's resulting pericenter radius is greater than the radius of the assisting planet, including the height of its atmosphere, $r_{p}>\left(R_{\mathrm{pl}}+h_{\mathrm{atm}}\right)$. Finally, to perform the gravity-assist computations, $\mathcal{R}$ is defined as in Appendix $\underline{\text { A. }}$

\section{Low-Thrust Trajectory Optimization Using Evolutionary Neurocontrollers}

The LTGA trajectory-optimization problem is attacked from the perspective of artificial intelligence and machine learning. In this context, a trajectory can be regarded as the result of a spacecraft steering strategy $\mathbf{S}$ that maps the problem-relevant variables $\mathbf{X} \in \mathcal{X}$ onto the spacecraft control vector $\mathbf{u} \in \mathcal{U}$ :

$$
\mathrm{S}: \mathcal{X}=\left\{\mathbf{x}_{\mathrm{sc}}, \mathbf{x}_{T}, \mathbf{x}_{\mathrm{pl}, i}, \mathbf{x}_{\mathrm{sc}}-\mathbf{x}_{T}, \mathbf{x}_{\mathrm{sc}}-\mathbf{x}_{\mathrm{pl}}, m_{p}\right\} \rightarrow \mathcal{U}=\{\mathbf{u}\}
$$

where $\mathbf{x}_{\mathrm{pl}, i}\left(i \in n_{\mathrm{pl}}\right)$ refers to the state of all $n_{\mathrm{pl}}$ potential gravityassisting bodies and $m_{p}$ is the actual propellant mass. Note that time is not explicitly a problem-relevant variable, because independent of time, the same constellation of planetary bodies requires the same steering vector. This way, the problem of searching the optimal spacecraft trajectory is equivalent to the problem of searching for (or learning) the optimal steering strategy $\mathrm{S}^{\star}$.

An artificial neural network (ANN) is used as a so-called neurocontroller (NC) to implement such spacecraft steering strategies. It can be considered as a parameterized function $\mathrm{N}$ (called network function) that is completely defined by the set of the network's internal parameters $\pi \in \mathbb{R}^{n_{\pi}}$. This way, each $\pi$ defines a steering strategy $\mathrm{S}_{\pi}$. The problem of searching for the optimal spacecraft trajectory is thus equivalent to the problem of searching for the optimal parameter set $\boldsymbol{\pi}^{\star}$, given a neurocontroller topology. Here, only feedforward ANNs are considered, in which neurons are organized hierarchically in three layers: namely, the input, hidden, and output layers [9]. Every neuron implements a sigmoid transfer function [11]. Every neuron in the input layer accepts one component of $\mathcal{X}$ as input, and the output layer consists of three neurons providing the spacecraft control vector $\mathbf{u}=\mathbf{u}(\gamma, \delta, \chi)$. The middle layer comprises 30 neurons, though the number of neurons in this layer generally has little effect on the optimality of the final steering strategy that the NC represents.

Evolutionary algorithms (EAs) that work on a population of strings can be used to find the optimal network parameters because they can be mapped on a string $\boldsymbol{\xi}$ (also called a chromosome or individual). The trajectory-optimization problem is then solved when the optimal chromosome $\xi^{\star}$ is found. Figure 3 summarizes the subsequent transformations of the optimal chromosome into the optimal trajectory. A neurocontroller that employs an evolutionary algorithm to learn the optimal control strategy is referred to as an evolutionary neurocontroller. Evolutionary operators that are tailored to work on real-valued strings and neural networks have been used: namely, one-point crossover operator, uniform crossover, crossover nodes, and mutation [11]. The quality of the solution and, even more so, the search duration depend on the population size. A good compromise between these two factors was found by selecting a population size of 50 individuals, the quality of the solution being quite insensitive to the actual number. A more detailed description of the implemented EA is beyond the scope of this paper and can be found in [11].

\section{A. InTrance}

The ENC architecture used in this work is sketched in Fig. 4. This particular ENC design reflects its application to solve LT trajectories optimization problems. To do so, the ENC runs in two loops. Within the inner trajectory-integration loop, a NC steers the spacecraft according to its network function $\mathrm{N}_{\pi}$ provided by the EA (that runs in the outer loop). The NC's parameter set $\pi$, which represents an individual, is therefore constant during integration. It can be thought of as a pilot who steers the spacecraft until the termination condition is met. That is, the pilot has either reached the final target or a maximum travel time (defined by the user). In the outer optimization loop, the EA holds a population $\Xi=\left\{\pi_{1}, \pi_{2}, \ldots, \pi_{q}\right\}$ of pilots (or chromosomes/individuals). The EA evaluates all pilots (i.e., NC parameter sets $\boldsymbol{\pi}_{j \in\{1, \ldots, q\}}$ ), one at a time, for their suitability to generate an optimal trajectory. Within the trajectory-optimization loop, the NC takes the actual spacecraft state $\mathbf{x}_{\mathrm{sc}}\left(\bar{t}_{i}\right)$ and that of the target body $\mathbf{x}_{T}\left(\bar{t}_{i}\right)$ as input values and maps them onto the output values from which the spacecraft control vector $\mathbf{u}\left(\bar{t}_{i}\right)$ can be calculated. At this point, $\mathbf{x}_{\mathrm{sc}}\left(\bar{t}_{i}\right)$ and $\mathbf{u}\left(\bar{t}_{i}\right)$ are inserted into the equations of motion, which are numerically integrated over one time step $\Delta \bar{t}=\bar{t}_{i+1}-\bar{t}_{i}$ to yield $\mathbf{x}_{\mathrm{sc}}\left(\bar{t}_{i+1}\right)$. This state is fed back into the NC. Again, the trajectory-integration loop stops when the termination condition is met. At this time, the NC's parameter set (i.e., its trajectory) is rated by the EA's fitness function $\mathrm{J}\left(\boldsymbol{\pi}_{j}\right)$. This fitness value is crucial to the reproduction probability of $\boldsymbol{\pi}_{j}$. Under the selection pressure of the environment, the EA breeds NCs that generate increasingly suitable steering strategies (offspring) that in turn generate increasingly better trajectories. The EA that is used within InTrance finally converges to a single steering strategy that gives, in the best case, a globally optimal trajectory $\mathbf{x}_{\mathrm{sc}}^{\star}[t]$.

\section{B. Objective Function}

The optimality of a trajectory can be defined with respect to different (primary) objectives such as transfer time or propellant consumption. When an ENC is used for trajectory optimization, the trajectory accuracy to the terminal constraints must also be considered as a secondary optimization objective, as they are not explicitly stated elsewhere. By defining a maximal allowed distance $\Delta r_{f, \max }$ and relative velocity $\Delta v_{f, \text { max }}$ at the target, the trajectory accuracy can be defined as follows with respect to the terminal constraints:

$$
\Delta X_{f}=\sqrt{\frac{1}{2}\left(\Delta R_{f}^{2}+\Delta V_{f}^{2}\right)}
$$

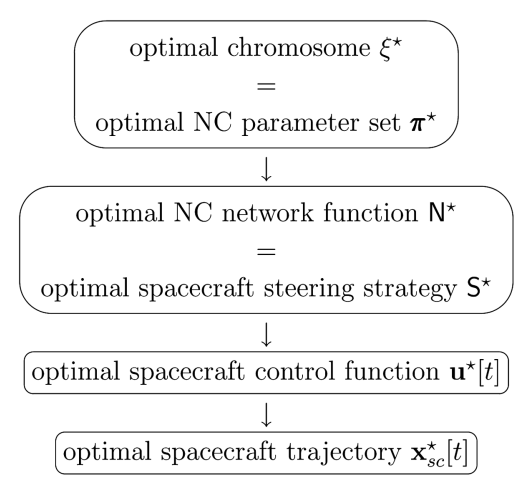

Fig. 3 From chromosome to optimal trajectory. 


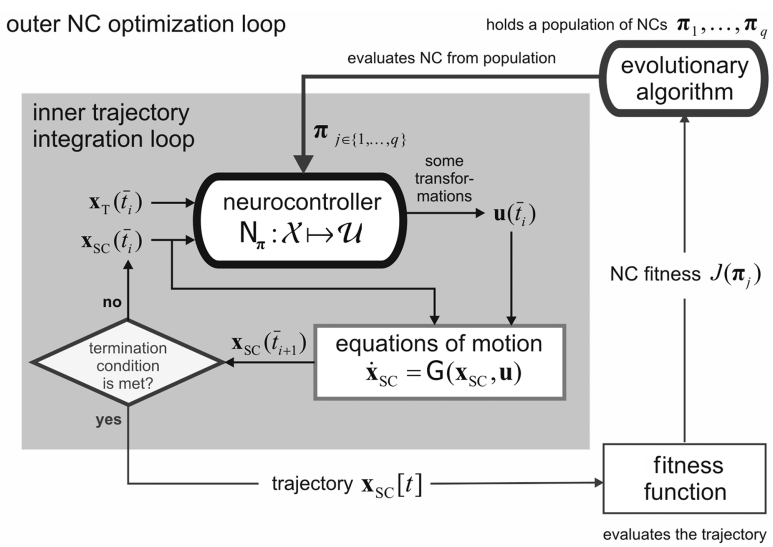

Fig. 4 Evolutionary neurocontrol architecture.

where

$$
\Delta R_{f}=\frac{\Delta r_{f}}{\Delta r_{f, \max }}
$$

and

$$
\Delta V_{f}=\frac{\Delta v_{f}}{\Delta v_{f, \max }}
$$

In the beginning of the search process, most individuals do not achieve the required accuracy, and hence a maximum transfer time $T_{\max }$ must be defined for the numerical integration of the trajectory. The subfitness functions

$$
\mathrm{J}_{T}=c_{1} \cdot\left(1-\frac{T}{T_{\max }}\right) \quad \mathrm{J}_{m_{p}}=\frac{m_{p}\left(\bar{t}_{0}\right)}{c_{2} m_{p}\left(\bar{t}_{0}\right)-m_{p}\left(\bar{t}_{f}\right)}-c_{3}
$$

(we have chosen $c_{1}=1000, c_{2}=2$, and $c_{3}=1 / 3$ ) for the primary optimization objectives have been found to work well and the subfitness functions

$$
\mathrm{J}_{r}=\log \frac{1}{\Delta R_{f}} \quad \mathrm{~J}_{v}=\log \frac{1}{\Delta V_{f}}
$$

have been defined for the secondary optimization objectives. $J_{r}$ and $J_{v}$ are positive if the respective accuracy requirement is fulfilled and they are negative if it is not. The values of the constants are arbitrary and do not influence the selection probability or the optimization result, because a tournament-selection procedure is used, in which the selection probability is independent of the scaling of the subfitness functions and the fitness function [11]. Simulations have showed that the search process should first concentrate on the accuracy of the trajectory and then on the primary optimization objectives. Therefore, the subfitness functions for the primary optimization objectives are modified to

$$
\begin{aligned}
& \mathrm{J}_{T}^{\prime}=\left\{\begin{array}{lll}
0 & \text { if } \mathrm{J}_{r}<0 & \text { or } \quad \mathrm{J}_{v}<0 \\
\mathrm{~J}_{T} & \text { if } \mathrm{J}_{r} \geq 0 & \text { and } \quad \mathrm{J}_{v} \geq 0
\end{array}\right. \\
& \mathrm{J}_{m_{p}}^{\prime}=\left\{\begin{array}{lll}
0 & \text { if } \mathrm{J}_{r}<0 & \text { or } \quad \mathrm{J}_{v}<0 \\
\mathrm{~J}_{m_{p}} & \text { if } \mathrm{J}_{r} \geq 0 & \text { and } \quad \mathrm{J}_{v} \geq 0
\end{array}\right.
\end{aligned}
$$

Therefore, to minimize the transfer time $T$ for a rendezvous, the following function is used:

$$
\mathrm{J}\left(T, \Delta r_{f}, \Delta v_{f}\right)=\mathrm{J}_{T}^{\prime}+\frac{1}{c_{4}+\left(1-c_{4}\right) \Delta X_{f}}
$$

(we have chosen $c_{4}=0.99$ ), whereas to minimize the propellant consumption $\Delta m_{p}$, the fitness function is similar, but $\mathrm{J}_{T}^{\prime}$ is replaced with $\mathrm{J}_{m_{p}}^{\prime}$ :

$$
\mathrm{J}\left(\Delta m_{p}, \Delta r_{f}, \Delta v_{f}\right)=\mathrm{J}_{m_{p}}^{\prime}+\frac{1}{c_{4}+\left(1-c_{4}\right) \Delta X_{f}}
$$

A tournament-selection scheme has been used for the selection of the individuals from the population [11]. This way, the selection probability does not depend on the scaling of the fitness function. The value chosen for $c_{4}$ guarantees that once the final constraints are fulfilled, improvements in the primary optimization objective are rated much higher than improvements in the fulfillment of the final constraints (but if two individuals have the same flight time or propellant consumption, the more accurate one is always preferred).

In the case of a planetary flyby, only the constraint on the position must be met, whereas the final velocity is set free. If the transfer time is to be minimized in this case, then

$$
\mathrm{J}\left(T, \Delta r_{f}\right)=\mathrm{J}_{m_{p}}^{\prime}+\frac{1}{c_{4}+\left(1-c_{4}\right) \Delta R_{f}}
$$

To minimize the propellant mass for a flyby, $\mathrm{J}_{T}^{\prime}$ is replaced with $\mathrm{J}_{m_{p}}^{\prime}$ :

$$
\mathrm{J}\left(\Delta m_{p}, \Delta r_{f}\right)=\mathrm{J}_{m_{p}}^{\prime}+\frac{1}{c_{4}+\left(1-c_{4}\right) \Delta R_{f}}
$$

\section{ENC Architecture Options}

Two different approaches to solve the LTGA problem are straightforward. In one case, the whole trajectory (i.e., the inbound leg from the departure point to the flyby body and the outbound leg from the flyby body to the target body) is optimized by a single NC. This choice is quite straightforward, as it requires only the input to the ANN and the chromosome length to be modified, but it preserves the structure of the EA operators. However, in such an approach, there is no objective evidence that the ENC will actually seek for gravityassist maneuvers and exploit them to gain orbital energy (only some expectations based on the ENC ability to perform solar photonic assists for solar sail trajectories [14]).

A second approach instead requires the use of multiple ENCs. Here, each ENC optimizes only a single leg of the trajectory. In other words, the mission is divided into sequential phases: the first phase starts from the departure body and ends at the SOI of the first flyby body. The gravity-assist maneuver at this moment is computed analytically to give the outbound conditions (subscript 2), then the second leg is optimized by a second ENC until the next SOI or the final target body is reached. This reasoning can be extended to $n+1$ ENCs $\left(\boldsymbol{\Psi}_{\mathbf{j} \in\{\mathbf{1}, \ldots, \mathbf{n}+\mathbf{1}\}}\right)$ performing $n$ gravity-assist maneuvers. At first sight, this second approach seems very promising because InTrance has proven to be capable of finding optimal $\boldsymbol{\Psi}_{\mathrm{j}}^{\star}$. By creating new evolutionary operators and providing a flyby sequence, one can be quite confident of finding the optimal overall trajectory for this sequence $\boldsymbol{\Psi}_{\mathbf{1}}^{\star}+\cdots+\boldsymbol{\Psi}_{\mathbf{n}+\mathbf{1}}^{\star}$. Although the latter approach is quite attractive, it fails in one particular point that is the goal of this work: designing a fully automatic tool that does not require the attendance of a trajectory-optimization expert. If the flyby sequence were to be given as an input to InTrance, either an expert or a second ad hoc algorithm would have to provide it. For this reason, the single-ENC approach has been chosen.

\section{Gravity-Assist Optimization}

Within the single-ENC approach, the first step is to provide the ENC with the appropriate input information. This corresponds to the knowledge the ENC should have to optimally steer the spacecraft and eventually perform gravity assists. In addition to the state of the spacecraft and the final target, the ENC should also know where the possible gravity-assist bodies are and how they are moving. In other words, the NC's inputs must include additional information such as $\mathbf{x}_{\mathrm{sc}}-\mathbf{x}_{\mathrm{pl}}$.

The second step is to introduce the analytical gravity-assist calculator into the inner trajectory-integration loop of the ENC. Here, a patched-conics approximation is used: at every integration step, a 


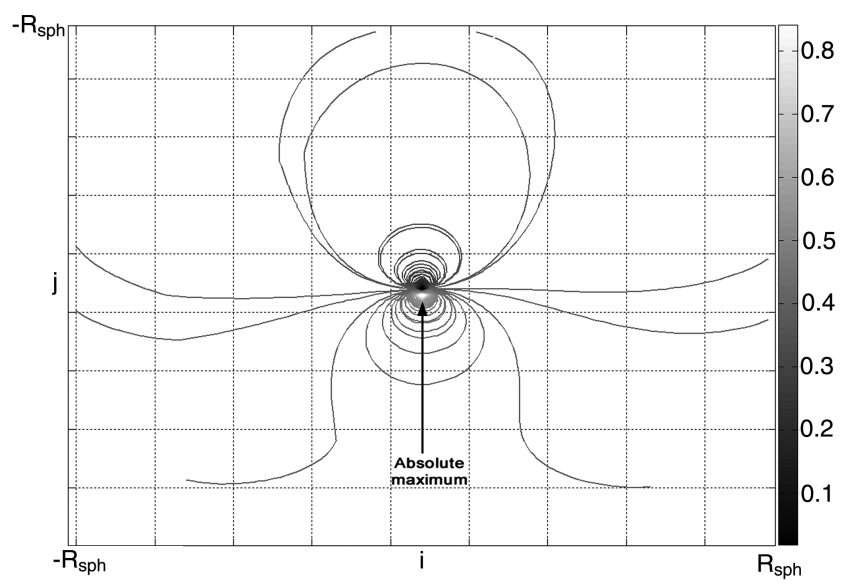

Fig. 5 Two-dimensional plot of $B$-plane fitness topology.

check is performed to determine whether the spacecraft has entered the SOI of a gravity-assist body. If this is the case, the maneuver is calculated analytically and the outbound conditions (subscript 2 ) are used as initial conditions for the successive numerical integration, which is carried out until the termination condition is met.

Finally, the third step corresponds to the gravity-assist maneuver optimization. To do so, the $B$ plane, which can be thought of as a target attached to the assisting body, is taken as reference system. The topology of the solution space for the optimal planetary maneuver was assessed by plotting the fitness values corresponding to a large-enough number of targeting points $(\bar{x}, \bar{y})$ on the $B$ plane for one exemplary gravity assist. Once a grid is defined on the plane, some virtual spacecraft were used to probe the solution space by forcing them to aim at each grid point. This way, a sufficient subset of all possible maneuvers was tested and rated with respect to the fitness function. Following this procedure, a plot can be made in which the $x$ and $y$ axes correspond to the $B$-plane coordinates, and the $z$ axis (the height of the surface) corresponds to the fitness value $\mathrm{J}_{(\bar{x}, \bar{y})}$. Figures $\underline{5}$ and 6 show that the solution space for this exemplary gravity assist is particularly smooth and regular (here, the Venus gravity assist of the test case in Sec. VIII is used).

For this exemplary gravity assist, the two extremes (i.e., the absolute maximum and the absolute minimum) are very well defined, and only a local minimum and maximum are present on the boundary of the SOI, because of the slight inclination of the fitness plane toward the positive $x$ axis. A few characteristics of the solution space are noteworthy:

1) As the gravity-assist geometry is incorporated in the neurocontroller's steering strategy (that is fixed once a neurocontroller is selected because its parameters are constant), each ENC can only fly one of the many possible GA geometries. Thus, the $B$ plane corresponds to a fixed point in space with a frozen incoming velocity vector (only the thrust profile may vary before and after the GA, but not the geometry of the flyby) characterized by a solution space with a single optimum.

2) The two global extremes are symmetrically located with respect to the origin and separated by about the diameter of the planet. There is in fact only one semi- $B$ plane that bends the spacecraft trajectory toward the target body, hence boosting the spacecraft in the right direction. Also, the two extremes are close to the flyby body in which its gravitational field is strongest and the trajectory's curvature is most affected.

3) The solution space in the outer regions is almost flat. This is also an expected result because the maneuvers performed close to the SOI

\footnotetext{
${ }^{\S}$ It is a planar coordinate system that contains the focus of the hyperbola and is perpendicular to the incoming asymptote. The origin of the reference system is the center of the assisting body, the $x$ axis is defined by the intersection of the $B$ plane with the trajectory plane, and the $y$ axis is orthogonal to the $x$ axis.
}

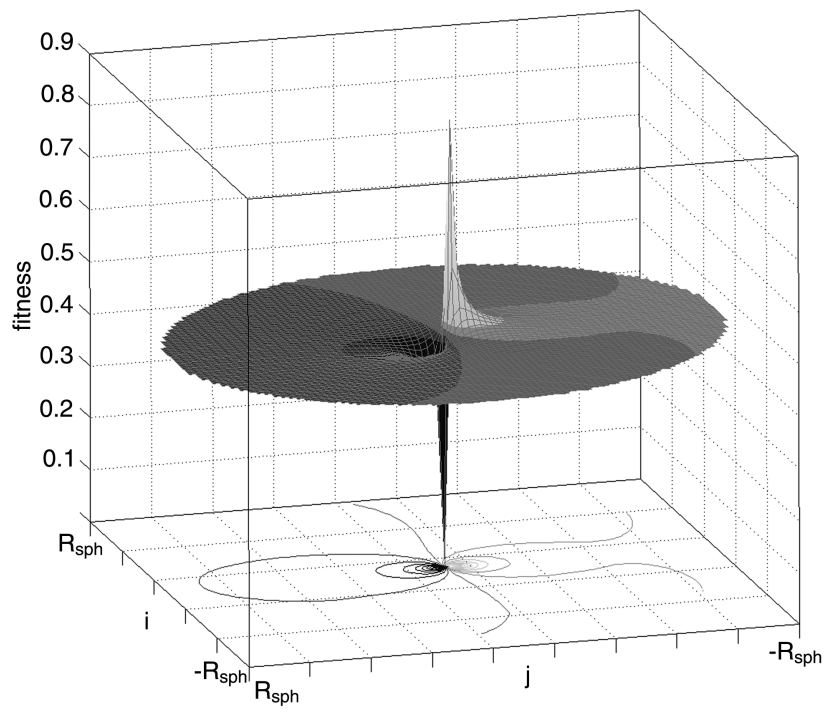

Fig. 6 Three-dimensional plot of $B$-plane topology.

boundaries (outer region of the equatorial circular plane in Fig. 5) have small effect on the trajectory.

4) No other hills or valleys are found in the domain. This suggests adopting a local optimization method rather than a global optimizer for the maneuver, for the sake of saving computing time. This simple structure of the solution space, however, might not generally be the case. Especially if multiple gravity assists are involved, a global method might offer advantages.

5) In this particular case, the fitness values are in the range $0<\mathrm{J}<0.9$ (note that $\mathrm{J} \geq 1$ corresponds to fulfillment of the final constraints), but the values close to the optimum are located in a very small region of the domain space.

Following the preceding analysis, we have chosen a steepestascent method as the optimization algorithm. The goal is to find the highest fitness-function value: that is, the highest reward $J$ that can be attributed to the navigator. Because local optima are present only in the outer regions of the SOI, there is no risk of converging to a local optimal solution if the initial point $\left(x_{i}, y_{i}\right)$ is chosen to be close enough to the planet. The strategy, which consists of following the path along the steepest gradient, is illustrated in Fig. 7. Using this simple algorithm, an efficient navigator for the ENC is designed. The best gravity-assist maneuver $(x, y)^{\star}$ is located within a few iterations (see Fig. 8) and is so efficient that the fitness of individuals that perform a gravity assist far outweighs the fitness of the individuals who do not.

The gravity-assist maneuver is performed when the spacecraft reaches the SOI of an assisting body. Here, the trajectory integration is stopped and the spacecraft's velocity vector defines the orientation of the $B$ plane. At this stage, the actual aiming point $\mathbf{r}_{1}$ is disregarded and the coordinate set $(x, y)^{\star}$ optimized by the navigator is used instead. This point $\check{\mathbf{r}}_{1}$ is projected onto the SOI to provide the initial

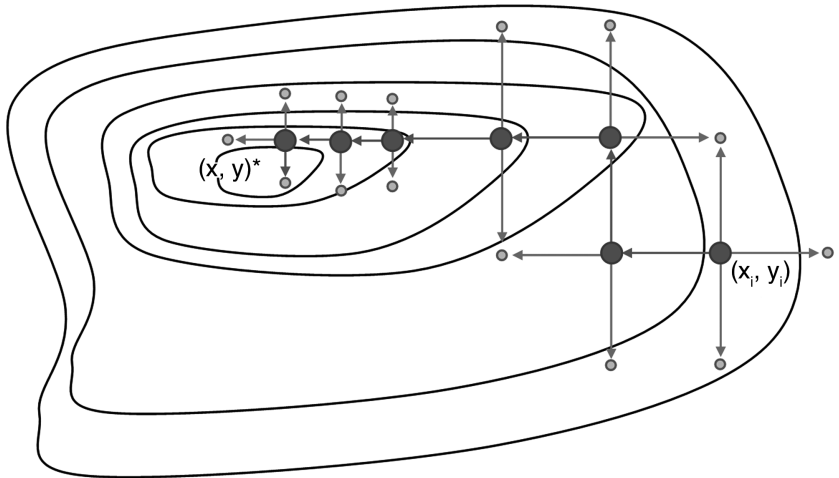

Fig. 7 Search algorithm. 


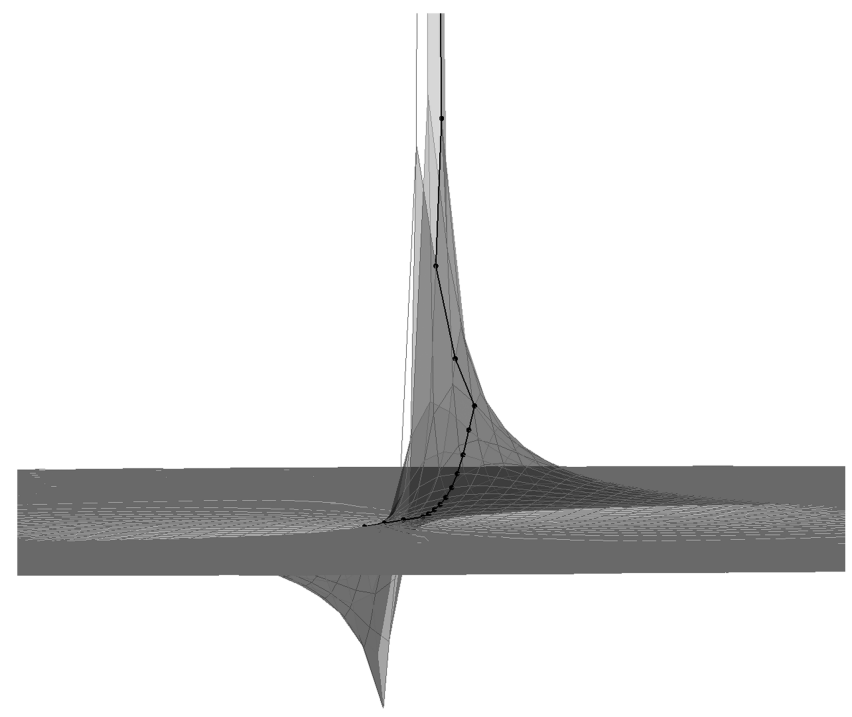

Fig. 8 Path followed by the steepest-ascent method.

position for the analytical gravity-assist calculator. The discontinuity that is introduced is minimized during the optimization process. This is done by introducing the distance $\left|\mathbf{r}_{1}-\check{\mathbf{r}}_{1}\right|$ in a subfitness function

$$
\mathrm{J}_{\text {distance }}=\frac{1}{\left|\mathbf{r}_{1}-\check{\mathbf{r}}_{1}\right|}
$$

that is to be maximized once the primary goal is achieved [ $J_{r} \geq 0$ and $J_{v} \geq 0$, as defined in Eq. (23)]. Finally, the gravity-assist maneuver is computed and the initial conditions for the following trajectory leg are obtained.

Because of the modest size of the SOIs, the chances for an ENC to encounter a sphere of influence are very small. To solve this problem, artificial spheres of influence are introduced to enlarge the real SOI sizes (with the exception of Jupiter and Saturn) by a factor $\alpha>1$. The idea is to let the ENC learn in an easier environment and gradually raise the difficulty by letting $\alpha \rightarrow 1$ while the number of reproductions $v$ in the population increases. Empirically, the following definition of $\alpha$ was found to provide a good learning environment to the ENC:

$$
\alpha= \begin{cases}\left(\frac{160+0.5 v}{40+0.5 v}\right) & \text { if } v<600 \\ 1 & \text { if } v \geq 600\end{cases}
$$

The 600th-generation threshold is chosen to provide the ENC with the real dynamic environment, typically after the primary goals are achieved and before the secondary goals are optimized. Note that the number of generations required is problem-dependent. However, no LTGA optimization problem was found to require less than $\sim 3000$ generations.

\section{Test Cases}

The algorithm defined in the previous section proved to be an efficient navigator for the ENC. The idea is to let the ENC (pilot) concentrate only on the trajectory-optimization task and support each pilot with a navigator whom he can consult whenever a SOI is crossed. Two missions are used as a reference to assess the validity of this method: a flyby mission to Pluto with a Jupiter gravity assist and a rendezvous mission to Mercury with a Venus gravity assist. The first mission is somewhat easier due to the very large size of Jupiter's SOI. The second mission is much more challenging due to the demanding $\Delta V$ requirement to reach Mercury.

\section{A. New Horizon Mission to Pluto}

The proposed approach was implemented in an extended version of InTrance called InTrance-GA. To assess its ability to optimize LTGA trajectories, a flyby mission to Pluto calculated by Vasile [ㅁ] was chosen as a reference. In that paper, the design of the NEP trajectories was performed with a direct transcription method based on finite elements in time (implemented in a software tool called DITAN $[15,16])$. However, because the problem presents a number of possible solutions dependent on the launch window and transfer time, a global optimization strategy was used [8] to generate sets of promising initial guesses. By setting the launch date and escape velocity to those used in the reference and the terminal conditions so that the arrival at Pluto is before 2020, InTrance-GA could generate results that are comparable with the so-called fast transit option in the reference. This was most probably achieved due to a very short integration step (i.e., one day). Thus, the objective of this simulation was not to optimize the launch conditions and find a global optimum solution, rather to explore the local level of accuracy that can be reached once a solution is found. The results summarized in Table 1 were obtained by evolving an initial population size of 50 individuals for 1367 generations, requiring a total computation time of 23,795 s and reaching an fitness-function value of $\mathrm{J}=33.77$. As can be seen, there is very good agreement between the two solutions. In particular, the propellant mass consumptions are nearly identical (the NEP model described in Sec. II.A. was used). Note that InTrance-GA was able achieve a very good local convergence so that virtually no refinement of the solution was needed. However, a minimum final flyby distance at Pluto as high as $\Delta r_{f}=1 e 6 \mathrm{~km}$ was reached and could not be further minimized, whereas $J_{\text {distance }}=(1755 \mathrm{~km})^{-1}$ was considered sufficiently low to consider the overall solution to be accurate.

\section{B. Mercury Rendezvous}

As a second test case to assess our method's performance, a rendezvous trajectory to Mercury, as calculated by Debban et al. [17] and De Pascale [18], was chosen. This optimization goal is particularly challenging for several reasons, including Mercury's proximity to the sun and the eccentricity and inclination of its orbit. Even assuming circular coplanar orbits of both Earth and Mercury, a Hohmann transfer requires a launch $V_{\infty}$ of at least $7.5 \mathrm{~km} / \mathrm{s}$, with the resulting arrival $V_{\infty}$ as high as $9.6 \mathrm{~km} / \mathrm{s}$ [19]. The idea of using chemical propulsion to remove this hyperbolic excess velocity is prohibitive, and hence LTGA techniques offer lower propellant requirements.

In the paper by Debban et al. [17], the whole computational effort is focused on the search for a proper initial guess [using the Satellite Tour Design Program (STOUR)-LTGA, a shape-based method that implements exponential sinusoid functions], which is then optimized using a direct method (implemented in a software tool called GALLOP). The chosen trajectory shape is a two-dimensional curve in polar coordinates. Here, the target's out-of-plane position at the time of the in-plane encounter is matched by using additional thrust acceleration acting along or against the spacecraft's angular momentum vector for some final portion of the leg's thrust arc. By assuming constant $I_{\mathrm{sp}}$, the propellant consumption is then estimated as a fraction of the initial spacecraft mass based on the time integral of the thrust acceleration and the rocket equation. Finally, the effect on the time of flight (TOF) is neglected. Thus, the trajectory along the $z$ coordinate is well approximated only for small inclination changes. An evolution of this method (implemented in a software tool called IMAGO) by De Pascale [1] describes the trajectory by assigning a

Table 1 Earth-Jupiter-Pluto mission with NEP

\begin{tabular}{lcc}
\hline \hline & DITAN & InTrance-GA \\
\hline Earth launch date & 19 January 2006 & 19 January 2006 \\
Launch $V_{\infty}$ & $12 \mathrm{~km} / \mathrm{s}$ & $12 \mathrm{~km} / \mathrm{s}$ \\
Mass at departure & $600 \mathrm{~kg}$ & $600 \mathrm{~kg}$ \\
Mass at arrival & $565.5 \mathrm{~kg}$ & $565.3 \mathrm{~kg}$ \\
$I_{\mathrm{sp}}$ & $3000 \mathrm{~s}$ & $3000 \mathrm{~s}$ \\
Thrust & $40 \mathrm{mN}$ & $40 \mathrm{mN}$ \\
Jupiter encounter & 23 February 2007 & 4 March 2007 \\
Pluto arrival & 4 October 2014 & 13 October 2014 \\
\hline \hline
\end{tabular}


Table 2 Earth-Venus-Mercury rendezvous trajectory

\begin{tabular}{lccc}
\hline \hline & GALLOP & IMAGO & InTrance-GA \\
\hline Earth launch date & 13 August 2002 & 1 July 2002 & 9 July 2002 \\
Launch $V_{\infty}$ & $1.93 \mathrm{~km} / \mathrm{s}$ & $3.0 \mathrm{~km} / \mathrm{s}$ & $2.99 \mathrm{~km} / \mathrm{s}$ \\
Propellant mass fraction & 0.310 & 0.3 & 0.314 \\
$I_{\text {sp }}$ & N/A & $3300 \mathrm{~s}$ & $3300 \mathrm{~s}$ \\
Earth-Venus TOF & 198 days & 183 days & 154 days \\
Total TOF & 853 days & 936 days & 1019 days \\
\hline \hline
\end{tabular}

set of shapes to a set of so-called pseudoequinoctial elements, allowing a fully three-dimensional description. These methods provide interesting results to assess the performance of InTranceGA.

The obtained results, together with the reference values, are shown in Table 2. Here, the SEP model as described in Sec. II.B was used, and the launch conditions were optimized over the following ranges: the launch date was between 15 June 2002 and 15 September 2002 and the launch $V_{\infty}$ range was set between 2 and $3 \mathrm{~km} / \mathrm{s}$. There is good agreement between InTrance-GA and IMAGO over the launch date, time of flight, and arrival date. The different SEP, trajectory model, and wider range of variability of the initial $V_{\infty}$ might instead justify the difference with GALLOP. InTrance-GA was able to rendezvous with Mercury after 2842 generations, requiring a total computation time of $41,484 \mathrm{~s}$, which once again demonstrates the complexity of this rendezvous problem. A final fitness value of $\mathrm{J}=973.4$ was reached, though the final rendezvous values were somewhat relaxed to $\Delta r_{f, \text { max }}=5 e 5 \mathrm{~km}$ and $\Delta v_{f, \max }=300 \mathrm{~m} / \mathrm{s}$. Once again, $J_{\text {distance }}$ proved to be an easier parameter to minimize, and a fairly low value of $(1230 \mathrm{~km})^{-1}$ was reached.

Figure 9 shows the trajectory found by InTrance-GA. The ENC manages to use the gravity-assist maneuver to obtain an initial orbitinclination change $\Delta \iota$ (the scale on the $z$ axis is exaggerated for the sake of visibility). The remaining inclination change $\Delta \iota=\iota_{\odot}-\iota_{\oplus}$ is carried out by the spacecraft's electric propulsion system. In addition to two correction maneuvers, the first immediately after departure and the second slightly before the Venus flyby, all thrust phases work to achieve the required orbit-inclination change.

The thrust and coast arcs can be better seen from Fig. 10, which provides a view from above the ecliptic plane, and from the spacecraft's throttle level shown in Fig. A1. Note that there are no clear switching points and the thrust is continuously throttled from 0 to its maximum. The solution is therefore expected to be locally suboptimal. Nevertheless, it represents a good first guess for this transfer trajectory.

\section{Conclusions}

We have presented a novel method (termed InTrance-GA) for low-thrust gravity-assist trajectory optimization, which is based on the use of evolutionary neurocontrollers. Based on an enumerative analysis of the solution space in the $B$ plane, a separate algorithm was

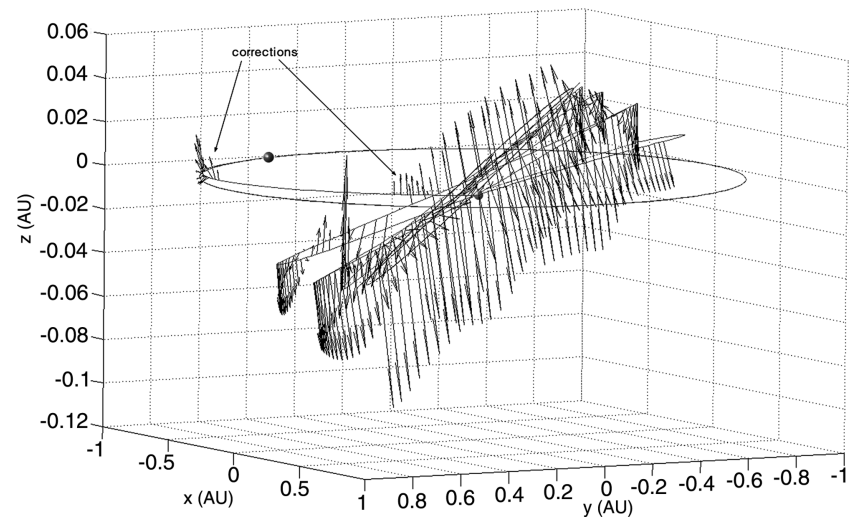

Fig. 9 Earth-Venus-Mercury trajectory and thrust vector ( $z$-axis scale exaggerated). implemented to optimize the gravity-assist maneuvers. This steepestascent algorithm acts as a navigator that the neurocontroller consults whenever it enters the sphere of influence of an assisting planet. Also, we have introduced artificially enlarged spheres of influence, due to their small size as compared with typical trajectory radii. This allows the evolutionary neurocontroller to learn in an easier environment, while gradually raising the difficulty by reducing the size to the real dimensions over time.

Preliminary results for a Pluto flyby with a Jupiter gravity assist and for a Mercury rendezvous with a Venus gravity assist have been presented. They show excellent agreement between the calculated solutions and the reference trajectories. In particular, in one of the

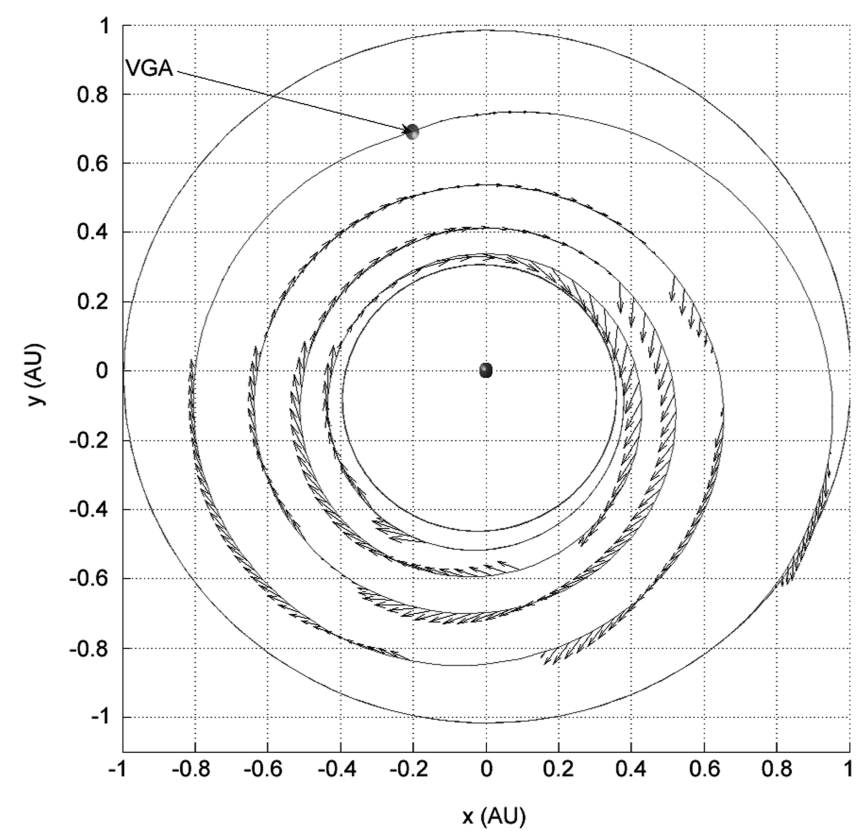

Fig. 10 Mercury rendezvous trajectory from above the ecliptic plane.

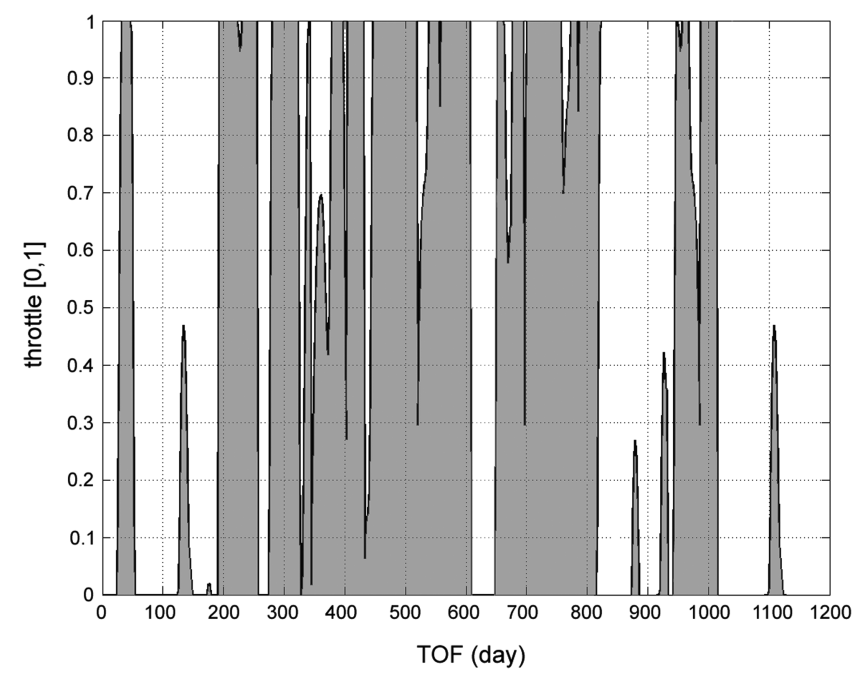

Fig. A1 Spacecraft's thrust history. 
cases, InTrance-GA was able to perform a global search of the solution space in a way that required virtually no further refinement of the solution.

\section{Appendix A: Gravity-Assist Model}

The rotation matrix $\mathcal{R}$, necessary to calculate the gravity-assist maneuver, is obtained by successive rotations: a first set of rotations transforms the Cartesian coordinate system into the polar orbital reference frame $\mathcal{O}$ defined by the three orthogonal unit vectors $\hat{\mathbf{e}}_{r}, \hat{\mathbf{e}}_{t}$, and $\hat{\mathbf{e}}_{h}$, where $\hat{\mathbf{e}}_{r}$ is along the radial sun-spacecraft direction, $\hat{\mathbf{e}}_{h}$ is along the spacecraft's orbital angular momentum vector, and the orbit transversal vector is $\hat{\mathbf{e}}_{t}=\hat{\mathbf{e}}_{h} \times \hat{\mathbf{e}}_{r}$. The peculiarity of this reference frame is that the $\hat{\mathbf{e}}_{t}$ axis changes constantly along the orbit. In other words, the angle $\zeta$ between $\hat{\mathbf{e}}_{t}$ and $\hat{\mathbf{e}}_{\varphi}$ equals the inclination of the orbit only at the nodes, whereas it is null at right angles. The value of this angle can be obtained using the spacecraft velocity vector expressed in polar coordinates $\left(v_{r}, v_{\varphi}, v_{\vartheta}\right)$ :

$$
\zeta=\arctan \frac{v_{\vartheta}}{v_{\varphi}}
$$

Thus, the first set of rotation matrices that transforms from the Cartesian into the polar orbital reference frame is obtained by three subsequent rotations about $\hat{\mathbf{e}}_{\theta}, \hat{\mathbf{e}}_{\varphi}$, and $\hat{\mathbf{e}}_{r}$. It follows that

$$
\mathcal{R}_{\text {pol,orb }}=\mathcal{R}_{r}\left(\zeta_{0}\right) \cdot \mathcal{R}_{\varphi}\left(-\vartheta_{0}\right) \cdot \mathcal{R}_{\vartheta}\left(\varphi_{0}\right)
$$

where $\varphi_{0}$ and $\vartheta_{0}$ represent the azimuth and elevation of the spacecraft at the moment it enters the SOI:

$$
\varphi_{0}=\arctan \frac{y}{x} \quad \vartheta_{0}=\arctan \frac{z}{\sqrt{x^{2}+y^{2}}}
$$

Once the reference frame is set to the polar orbital, the second rotation is the one that actually carries out the maneuver. Hence, the velocity and position vectors are rotated about the orbital angular momentum vector $\hat{\mathbf{e}}_{h}$ by, respectively, angles $\varphi$ and $\phi$ defined by Eq. (19) through the rotation matrix $\mathcal{R}_{\hat{\mathbf{e}}_{h}}$. Finally, a third set of rotations is used to bring the reference system back to Cartesian. These rotation matrices can be easily obtained by transposing Eq. (A2):

$$
\begin{gathered}
\mathcal{R}_{\text {cart }}=\mathcal{R}_{\text {pol,orb }}^{T}=\left(\mathcal{R}_{r}\left(\zeta_{0}\right) \cdot \mathcal{R}_{\varphi}\left(-\vartheta_{0}\right) \cdot \mathcal{R}_{\vartheta}\left(\varphi_{0}\right)\right)^{T} \\
=\mathcal{R}_{\vartheta}^{T}\left(\varphi_{0}\right) \cdot \mathcal{R}_{\varphi}^{T}\left(-\vartheta_{0}\right) \cdot \mathcal{R}_{r}^{T}\left(\zeta_{0}\right)
\end{gathered}
$$

All the subsequent rotation matrices have now been defined and the final expression for the overall rotation matrix $\mathcal{R}$ in Eqs. (17) and (18) can be provided:

$$
\begin{aligned}
& \mathcal{R}(\phi)=\mathcal{R}_{\text {cart }} \cdot \mathcal{R}_{\hat{\mathbf{e}}_{h}}(\phi) \cdot \mathcal{R}_{\mathrm{pol}, \mathrm{orb}}=\mathcal{R}_{\vartheta}^{T}\left(\varphi_{0}\right) \cdot \mathcal{R}_{\varphi}^{T}\left(-\vartheta_{0}\right) \cdot \mathcal{R}_{r}^{T}\left(\zeta_{0}\right) \\
& \cdot \mathcal{R}_{\hat{\mathbf{e}}_{h}}(\phi) \cdot \mathcal{R}_{r}\left(\zeta_{0}\right) \cdot \mathcal{R}_{\varphi}\left(-\vartheta_{0}\right) \cdot \mathcal{R}_{\vartheta}\left(\varphi_{0}\right) \\
& \mathcal{R}(\varphi)=\mathcal{R}_{\text {cart }} \cdot \mathcal{R}_{\hat{\mathbf{e}}_{h}}(\varphi) \cdot \mathcal{R}_{\mathrm{pol}, \mathrm{orb}}=\mathcal{R}_{\vartheta}^{T}\left(\varphi_{0}\right) \cdot \mathcal{R}_{\varphi}^{T}\left(-\vartheta_{0}\right) \cdot \mathcal{R}_{r}^{T}\left(\zeta_{0}\right) \\
& \cdot \mathcal{R}_{\hat{\mathbf{e}}_{h}}(\varphi) \cdot \mathcal{R}_{r}\left(\zeta_{0}\right) \cdot \mathcal{R}_{\varphi}\left(-\vartheta_{0}\right) \cdot \mathcal{R}_{\vartheta}\left(\varphi_{0}\right)
\end{aligned}
$$

Using Eqs. (17) and (18) together with Eqs. (A5) and (A6), the spacecraft's planetocentric Cartesian state after the maneuver is defined. Its components can be added to those of the assisting planet to define the spacecraft's location and velocity at the moment it leaves the SOI in the heliocentric ecliptic coordinate system:

$$
\begin{aligned}
& \mathbf{R}_{2}=\mathbf{r}_{2}+\mathbf{R}_{\mathrm{pl}, 2} \\
& \mathbf{V}_{2}=\mathbf{v}_{2}+\mathbf{V}_{\mathrm{pl}, 2}
\end{aligned}
$$

where subscript 2 is used to indicate a variable after the gravity assist has occurred (i.e., at time $t+\Delta t_{\mathrm{pl}}$ ), and $\mathbf{R}_{\mathrm{pl}, 2}$ and $\mathbf{V}_{\mathrm{pl}, 2}$ can be computed using the osculating elements of the body and the duration of the maneuver. The time of flight for a hyperbolic orbit within the
SOI is defined as

$$
\Delta t_{\mathrm{pl}}=2 \sqrt{\frac{a^{3}}{\mu}}\left(\frac{\sinh H}{\sin \delta}-H\right)
$$

where $H$ is the hyperbolic eccentric anomaly. Its value is determined by the semimajor axis $a$ of the hyperbola, the semirotation angle $\delta$, and the radius $R_{\mathrm{SOI}}$ of the $\mathrm{SOI}$ :

$$
H=\cosh ^{-1}\left[\left(1+\frac{R_{\mathrm{SOI}}}{a}\right) \sin \delta\right] \quad a=\left(\frac{\left|\mathbf{v}_{1}\right|^{2}}{\mu}-\frac{2}{R_{\mathrm{SOI}}}\right)^{-1}
$$

\section{Acknowledgment}

The authors would like to express their gratitude to Wolfgang Seboldt at DLR, German Aerospace Center, for always finding the time for suggestions and in-depth discussions.

\section{References}

[1] Racca, G. D., "Capability of Solar Electric Propulsion for Planetary Missions," Planetary and Space Science, Vol. 49, No. 14, Dec. 2001, pp. 1437-1444. doi:10.1016/S0032-0633(01)00084-8

[2] Vasile, M., and De Pascale, P., "Preliminary Design of Multiple Gravity-Assist Trajectories," Journal of Spacecraft and Rockets, Vol. 43, No. 4, 2006, pp. 794-805. doi: $10.2514 / 1.17413$

[3] Izzo, D., Becerra, V. M., Myatt, D. R., Nasuto, S. J., and Bishop, J. M., "Search Space Pruning and Global Optimisation of Multiple Gravity Assist Spacecraft Trajectories," Journal of Global Optimization, Vol. 38, June 2007, pp. 283-296. doi:10.1007/s10898-006-9106-02003.

[4] McConaghy, T., Debban, T., Petropoulos, A., and Longuski, J., "Design and Optimization of Low-Thrust Trajectories with Gravity Assists," Journal of Spacecraft and Rockets, Vol. 40, No. 3, 2003, pp. 380-387. doi: $10.2514 / 2.3973$

[5] Hartmann, J., "Low-Thrust Trajectory Optimization using Stochastic Optimization Techniques," M.S. Thesis, Univ. of Illinois at UrbanaChampaign, Urbana, IL, 1996.

[6] Coverstone-Carroll, V., Hartmann, J., and Mason, W., "Optimal MultiObjective Low-Thrust Spacecraft Trajectories," Computer Methods in Applied Mechanics and Engineering, Vol. 186, Nos. 2-4, June 2000, pp. 387-402.

doi:10.1016/S0045-7825(99)00393-X

[7] De Pascale, P., Vasile, M., and Ercoli Finzi, A., "A Tool for Preliminary Design of Low Thrust Gravity-Assist Trajectories," Space Flight Mechanics Conference, Maui, HI, American Astronautical Society Paper 04-250, Feb. 2004.

[8] Vasile, M., "A Global Approach to Optimal Space Trajectory Design," AAS/AIAA Space Flight Mechanics Meeting, Ponce, Puerto Rico, American Astronautical Society Paper 03-141, Feb. 2003.

[9] Dachwald, B., "Optimization of Interplanetary Solar Sailcraft Trajectories Using Evolutionary Neurocontrol," Journal of Guidance, Control, and Dynamics, Vol. 27, No. 1, 2004, pp. 66-72. doi:10.2514/1.9286

[10] Betts, J., "Practical Methods for Optimal Control Using Nonlinear Programming," Advances in Control and Design Series, Society for Industrial and Applied Mathematics, Philadelphia, 2001.

[11] Dachwald, B., "Low-Thrust Trajectory Optimization and Interplanetary Mission Analysis Using Evolutionary Neurocontrol," Ph.D. Thesis, Univ. der Bundeswehr München; Fakultät für Luft- und Raumfahrttechnik; Inst. für Raumfahrttechnik, Munich, 2004.

[12] Williams, S., and Coverstone-Carroll, V., "Mars Missions Using Solar Electric Propulsion," Journal of Spacecraft and Rockets, Vol. 37, No. 1, 2000, pp. 71-77. doi: $10.2514 / 2.3528$

[13] Labunsky, A., Papkov, O., and Sukhanov, K., Multiple Gravity Assist Interplanetary Trajectories, 1st ed., Gordon and Breach, Amsterdam, 1998, pp. 10-15.

[14] Dachwald, B., "Optimal Solar Sail Trajectories for Missions to the Outer Solar System," Journal of Guidance, Control, and Dynamics, Vol. 28, No. 6, 2005, pp. 1187-1193. doi: $10.2514 / 1.13301$ 
[15] Vasile, M., and Bernelli-Zazzera, F., "Targeting a Heliocentric Orbit Combining Low-Thrust Propulsion and Gravity Assist Manoeuvres," Operations Research in Space and Air, Applied Optimization, Vol. 79, Kluwer Academic, Norwell, MA, 2003.

[16] Vasile, M., and Bernelli-Zazzera, F., "Optimizing Low-Thrust and Gravity Assist Manoeuvres to Design Interplanetary Trajectories," Journal of the Astronautical Sciences, Vol. 51, No. 1, Jan.-Mar. 2003, pp. 13-35.

[17] Debban, T., Mc Conaghy, T., and Longuski, J., "Design and
Optimization of Low-Thrust Gravity-Assist Trajectories to Selected Planets," AIAA/AAS Astrodynamics Specialist Conference, Monterey, CA, AIAA Paper 2002-4729, Aug. 2002.

[18] De Pascale, P., "Strumenti di Indagine Globale per la Progettazione Preliminare di Traiettorie Interplanetarie," M.S. Thesis, Politecnico di Milano, Milano, Italy, 2003.

[19] Sims, J., "Delta-V Gravity Assist Trajectory Design: Theory and Practice," Ph.D. Thesis, School of Aeronautics and Astronautics, Purdue Univ., West Lafayette, IN, 1996. 